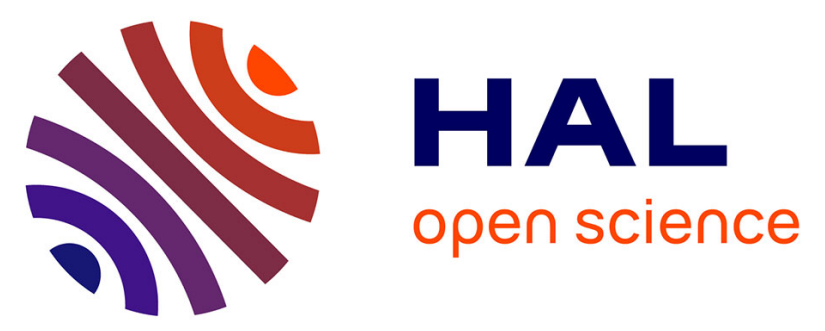

\title{
Assessing the effective elastic properties of the tendon-to-bone insertion: a multiscale modeling approach
}

\author{
A. Aghaei, N. Bochud, G. Rosi, S. Naili
}

\section{- To cite this version:}

A. Aghaei, N. Bochud, G. Rosi, S. Naili. Assessing the effective elastic properties of the tendonto-bone insertion: a multiscale modeling approach. Biomechanics and Modeling in Mechanobiology, 2021, 20 (2), pp.433-448. 10.1007/s10237-020-01392-7 . hal-03208707

\section{HAL Id: hal-03208707 \\ https://hal.science/hal-03208707}

Submitted on 26 Apr 2021

HAL is a multi-disciplinary open access archive for the deposit and dissemination of scientific research documents, whether they are published or not. The documents may come from teaching and research institutions in France or abroad, or from public or private research centers.
L'archive ouverte pluridisciplinaire $\mathbf{H A L}$, est destinée au dépôt et à la diffusion de documents scientifiques de niveau recherche, publiés ou non, émanant des établissements d'enseignement et de recherche français ou étrangers, des laboratoires publics ou privés. 


\title{
Assessing the effective elastic properties of the tendon-to-bone insertion: A multiscale modeling approach
}

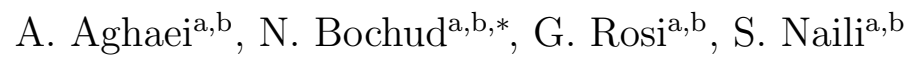 \\ ${ }^{a}$ Univ Paris Est Creteil, CNRS, MSME, F-94010 Creteil, France \\ ${ }^{b}$ Univ Gustave Eiffel, MSME, F-77454 Marne-la-Vallée, France
}

\begin{abstract}
The interphase joining tendon to bone plays the crucial role of integrating soft to hard tissues, by effectively transferring stresses across two tissues displaying a mismatch in mechanical properties of nearly two orders of magnitude. The outstanding mechanical properties of this interphase are attributed to its complex hierarchical structure, especially by means of competing gradients in mineral content and collagen fibers organization at different length scales. The goal of this study is to develop a multiscale model to describe how the tendon-to-bone insertion derives its overall mechanical behavior. To this end, the effective anisotropic stiffness tensor of the interphase is predicted by modeling its elastic response at different scales, spanning from the nanostructural to the mesostructural levels, using continuum micromechanics methods. The results obtained at a lower scale serve as inputs for the modeling at a higher scale. The obtained predictions are in good agreement with stochastic finite element simulations and experimental trends reported in literature. Such model has implication for the design of bioinspired bi-materials that display the functionally graded properties of the tendon-to-bone insertion.
\end{abstract}

Keywords: Tendon-to-bone insertion, Homogenization, Continuum micromechanics, Biological interphase, Functionally graded material, Partially mineralized tissue

${ }^{*}$ Corresponding author: nicolas.bochud@u-pec.fr 


\section{Introduction}

2 The interaction between soft and hard tissues is essential to ensure good mobility to the musculoskeletal system. Specifically, the integration between tendon (or ligament) and bone 4 occurs through a particular tissue interphase called enthesis, which derives from the ancient 5 Greek word referring to the insertion. According to their musculoskeletal site of insertion, entheses can be characterized as fibrous or fibrocartilaginous [1]. Fibrous (or indirect) insertions are usually found where tendons and ligaments attach to the shaft of long bones, such as the insertion of the deltoid tendon into the humerus or the tibial insertion of the medial collateral ligament. Fibrocartilaginous (or direct) insertions are more common and present at the bony attachments of the rotator cuff, the anterior cruciate ligament and the Achilles tendons. Fibrocartilaginous insertions are generally divided into four distinct regions with different compositions, functions, and biomechanical properties: (1) the tendon, which consists of aligned type I collagen fibers with a small amount of non-collagenous proteins (NCPs), including proteoglycans and the remaining volume filled with water [2]; (2) a non-mineralized fibrocartilaginous region, which is an avascular zone consisting of unmineralized types I, II and III collagen fibers and proteoglycan aggrecan [3]; (3) a mineralized fibrocartilaginous region, which is mostly characterized by partially mineralized type II collagen fibers, the proteoglycan aggrecan being produced by fibrocartilage cells or fibrochondrocytes between bundles of collagen fibers [4]; and (4) the bone, which is a composite material that mainly consists of highly mineralized type I collagen [5].

Although these different tissue regions are compositionally distinct, they are structurally continuous. Moreover, it is acknowledged that the mechanical environment of the enthesis is complex and heterogeneous, as it is subject to tensile, compressive and shear forces [6], in order to ensure an effective transfer of the mechanical stresses across the two surroundings tissues, which show a mismatch in mechanical properties of nearly two orders of magnitude. Three main features contribute to the mechanical effectiveness of the enthesis: (1) its geometry is characterized by a shallow attachment angle and an optimized shape of the outward splay, which improves its resistance to mechanical loads [7]; (2) the interdigitation of the 
bone within the tendon, described as a wave-like structure, allows a gain in toughness, as well as a better distribution of stresses on the fraction of tissue implied in this mechanism [8]; and (3) a compositional and structural gradient, through a gradual increase in mineral content together with a corresponding reorganization of the collagen fibers from the tendon to the bone [9]. The combination of these multiscale factors is hypothesized to lead to an intermediate zone more compliant than either tendon or bone, thus ensuring an effective distribution of mechanical stresses, which allows reducing the risk of rupture $[10,11]$.

Musculoskeletal injuries involving tendon rupture typically require surgical reattachment back to bone. Such operations exhibit a high rate of failure because the enthesis rarely regenerates and does not recover its mechanical properties. This clinical burden is mainly due to the lack of understanding of the healthy attachment, as well as of the damaging process that may occur at this interphase [12]. To date, studies on the tendon-to-bone insertion have mainly focused on the rehabilitation methods to be implemented after a tendon operation, as well as on the histology of the enthesis [13]. The targeted parameters generally included compositional and structural features at lower length scales (e.g., collagen types, organization and morphology of collagen fibers, degree of mineralization), but seldom the mechanical properties at the tissue scale. Indeed, the direct mechanical measurement of the tendon-to-bone insertion has been shown to be very complicated, particularly because of its heterogeneity and small dimensions (typically a few $\mu \mathrm{m}$ to some $\mathrm{mm}$ according to the insertion site) [10, 14]. The excellent mechanical properties of the enthesis are attributed to its complex hierarchical structure. Nonetheless, understanding how this hierarchy and the resulting mechanical properties at the different scales affect its overall mechanical behavior and allow such an effective stress transfer across this interphase represents a considerable challenge. To address this issue, tools such as multiscale modeling could help predicting the effective stiffness properties of the enthesis at the tissue level, as well as studying the individual impact of lower-scale features on its overall mechanical behavior at higher scales. Regardless of the considered medium -tendon, enthesis or bone-, all these regions substantially have a similar hierarchical collagen-based structure starting with collagen molecules at the nanoscale up to a pattern of collagen fibers surrounded by an extracellular matrix at 
higher scales [15]. Several analytical and computational models have been proposed to infer the mechanical properties of bone at different scales, in particular using homogenization methods [16-19]. In the same way, a few studies have been conducted on the hierarchical modeling of (mineralized) tendon [20, 21]. However, little information concerning the multiscale modeling of the tendon-to-bone insertion is available in the literature. Biomechanical models were initially proposed by considering the collagen-mineral interactions and the collagen fiber architecture at the microscale. In a pioneering study, Genin et al. [22] estimated the stiffness of partially mineralized fibers by randomly distributing minerals among unmineralized cylindrical collagen fibrils using Monte Carlo simulations, which subsequently allowed determining the isotropic mechanical properties of the partially mineralized tissue at the macroscale. Later, the same group introduced a progressive stiffening model for collagen, by accounting for different sequences of mineral accumulation across spatial hierarchy levels spanning from the fibril to the tissue level, to estimate the mechanical properties of partially mineralized collagen tissue [23]. In a subsequent study, they presented an estimate for composites containing multiple classes of aligned ellipsoidal inclusions with a relatively high volume fraction, which demonstrated its potential application to the graded tissue interphase at the tendon-to-bone attachment [24]. More recently, the micromechanical behavior of tendon-to-bone structures was studied and compared with a composite beam-bending model [25]. The beam was modeled as a three-phase composite in series, in which the modulus of the transitional graded interphase was defined as a linear extrapolation between those of the mineralized and unmineralized regions. All these models provided a general understanding on the mechanical properties of the insertion site (i.e., average Young modulus) at different structural levels. However, to the best of our knowledge, these models only partially accounted for the anisotropic nature of the interphase at the different scales, and the transition between different hierarchies from the nanoscale to the mesoscale was not achieved. Moreover, the impact of the individual nanoscale properties on the effective stiffness tensor at the mesoscale was not addressed so far.

To face these current limitations, this study aims at developing a hierarchical model to predict the effective anisotropic stiffness tensor of the tendon-to-bone insertion by model- 
ing its elastic response at different scales spanning from the nano- to the mesostructural levels. To this end, a continuum micromechanics approach was proposed by distinguishing six homogenization steps at four different scales. First, we derived the effective stiffness properties of the enthesis at the mesoscale by accounting for gradients in mineral content and collagen fibers organization across the interphase at lower scales. Second, we carried out a parametric study to evaluate the impact of the mechanical properties of each nanoscale component on the overall mechanical behavior of the insertion site. Third, based on the knowledge of the mechanical properties of the two surroundings tissues at the mesoscale, an optimization procedure was solved to identify the nanoscale properties that yield the best agreement between the model predictions and ultrasonic data reported in the literature. Numerical results show that the effective mechanical properties predicted by our multiscale model are in good agreement with earlier modeling approaches and experimentally observed trends. Moreover, our model is straighforward and could be readily extended to account for damage characteristics of the interphase as a footprint of clinical burden.

The paper is structured as follows: Sec. 2 recalls the fundamentals of micromechanicsbased models and introduces the proposed multiscale modeling of the enthesis. The model parameters, i.e., the mechanical properties, volume fractions and gradients of the tendonto-bone insertion's components, are then described in Sec. 3. Finally, the numerical results are presented in Sec. 4 and discussed in the light of the literature in Sec. 5.

\section{Methods}

In this section, a multiscale model of the tendon-to-bone insertion based on a hierarchical modeling approach that consists of successive homogenization steps from the nanostructural to the mesostructural levels is proposed. This multiscale model relies on several hypotheses: (1) whereas mineral grading can occur at any length scale, it was incorporated here at the nanoscale [26]; (2) the gradient in collagen fibers organization occured at the microscale [22]; (3) type I collagen was assumed to be the main organic component across the interphase; and (4) the mechanical properties of the elementary nanoscale components (collagen, hydroxyapatite (HA), water and NCPs), which were used as input data in our model, were all 
assumed to have a linear elastic and isotropic behavior.

\subsection{Fundamentals of micromechanics-based models}

Continuum micromechanics allows estimating the effective stiffness properties of heterogeneous materials [27], which can generally be derived by solving the matrix-inclusion problem according to Eshelby [28]. A basic concept of continuum micromechanics consists in selecting a representative volume element (RVE) in the structure. Based on the separation-of-scales requirement, the characteristic length $\ell$ of this RVE must be considerably larger than the dimension of the heterogeneities $d$ within the RVE and implicitly smaller than the characteristic length of the structure $\mathcal{L}$, so that $d \ll \ell \ll \mathcal{L}$. Useful information concerns volume fractions and elastic properties of phases, the existence of one connected matrix phase in which one or several inclusion phases with different shapes are embedded or the disordered arrangement of all phases. Hence, the homogenized stiffness tensor of such RVE, $\mathbb{C}_{\text {hom }}$, can be stated as in [29]

$$
\mathbb{C}_{\mathrm{hom}}=\sum_{r=1}^{n} f_{r} \mathbb{C}_{r}:\left[\mathbb{I}+\mathbb{P}_{r}^{0}:\left(\mathbb{C}_{r}-\mathbb{C}^{0}\right)\right]^{-1}:\left\{\sum_{s=1}^{n} f_{s}\left[\mathbb{I}+\mathbb{P}_{s}^{0}:\left(\mathbb{C}_{s}-\mathbb{C}^{0}\right)\right]^{-1}\right\}^{-1}
$$

where : denotes the double contracted product of two tensors, $n$ is the number of phases in the RVEs, $f_{r}$ denotes the volume fraction of the phase $r, \mathbb{I}$ is the fourth-order identity tensor, $\mathbb{C}_{r}$ is the stiffness tensor of phase $r, \mathbb{C}^{0}$ is the stiffness tensor of the matrix phase, and $\mathbb{P}^{0}$ is the so-called Hill tensor of phase $r$ embedded in the matrix phase, whose form depends on the shape of the inclusion and on the stiffness tensor of the surrounding matrix $\mathbb{C}^{0}$. The Hill tensor can be related to the Eshelby tensor as $\mathbb{P}^{0}=\mathbb{S}_{\text {Esh }}^{0}:\left(\mathbb{C}^{0}\right)^{-1}$. The components of the Hill tensors for the different inclusion shapes considered in this study are provided in Appendix A.

Several approaches for determining $\mathbb{C}^{0}$ have been proposed in the literature. Among these, two methods are particularly relevant to our context. First, the Mori-Tanaka scheme can be applied when the inclusion phase consists of small particles surrounded by a continuous homogeneous matrix [30]. In such a case, the matrix phase can be directly identified as the actual matrix phase $\left(\right.$ i.e, $\mathbb{C}^{0}=\mathbb{C}_{\text {matrix }}$ ). Second, when no matrix phase can be 
clearly identified, it is convenient to apply the self-consistent scheme [31]. The underlying hypothesis of this second method consists in estimating the homogenized properties of the effective medium by locating each inhomogeneity in the homogeneous effective medium (i.e, $\left.\mathbb{C}^{0}=\mathbb{C}_{\text {hom }}\right)$

\subsection{Multiscale modeling of the tendon-to-bone insertion}

According to earlier studies applied to partially mineralized tissues, such as bone $[17,32,33]$ and mineralized tendon [20], we hierarchically modeled here the mechanical behavior of the tendon-to-bone insertion by considering four relevant hierarchical levels compound of six homogenization steps (see Fig. 1), and by assuming gradients in mineral content and collagen fibers organization across the interphase between the two surroundings tissues [22, 23]. Across the enthesis, mineral crystals replace the water by filling the gap zones between collagen thereafter spreading through the fibrils surface [34]. Extrapolating this consideration to our modeling approach, the fibril array was considered as a hydrated collagen fibril at the nanoscale (i) without mineral particles in the tendon region, (ii) with a functional grading of mineral across the insertion, and (iii) as a partially mineralized collagen fibril in the bone region. The gradient of mineralization was introduced in the model by means of a normalized mineral volume fraction, $\phi(x)$, defined as the ratio of the overall mineral volume fraction $f_{\text {mineral }}(x)$ at a normalized position along the insertion, $x$, to the overall volume fraction of mineral at the bone level, $f_{\text {mineral }}^{\text {bone }}$, so that $\phi(x)=f_{\text {mineral }}(x) / f_{\text {mineral }}^{\text {bone }}$. The normalized position $x$ across the interphase was thus $x=0$ at the end of the tendon (i.e., $\phi(x)=0$ ) and $x=1$ at the beginning of the bone (i.e., $\phi(x)=1$ ), so that $\phi(x) \approx x$. Moreover, the gradient in collagen fibers organization was accounted for by considering the angular deviation of the collagen fibers distribution at a normalized position $x$ along the insertion [22]. At each scale, the homogenized stiffness tensor of the enthesis, $\mathbb{C}_{\text {hom }}(x)$, was therefore related to a precise description of the geometric and mechanical properties of each of the subscales at any normalized position $x$ across the interphase. 


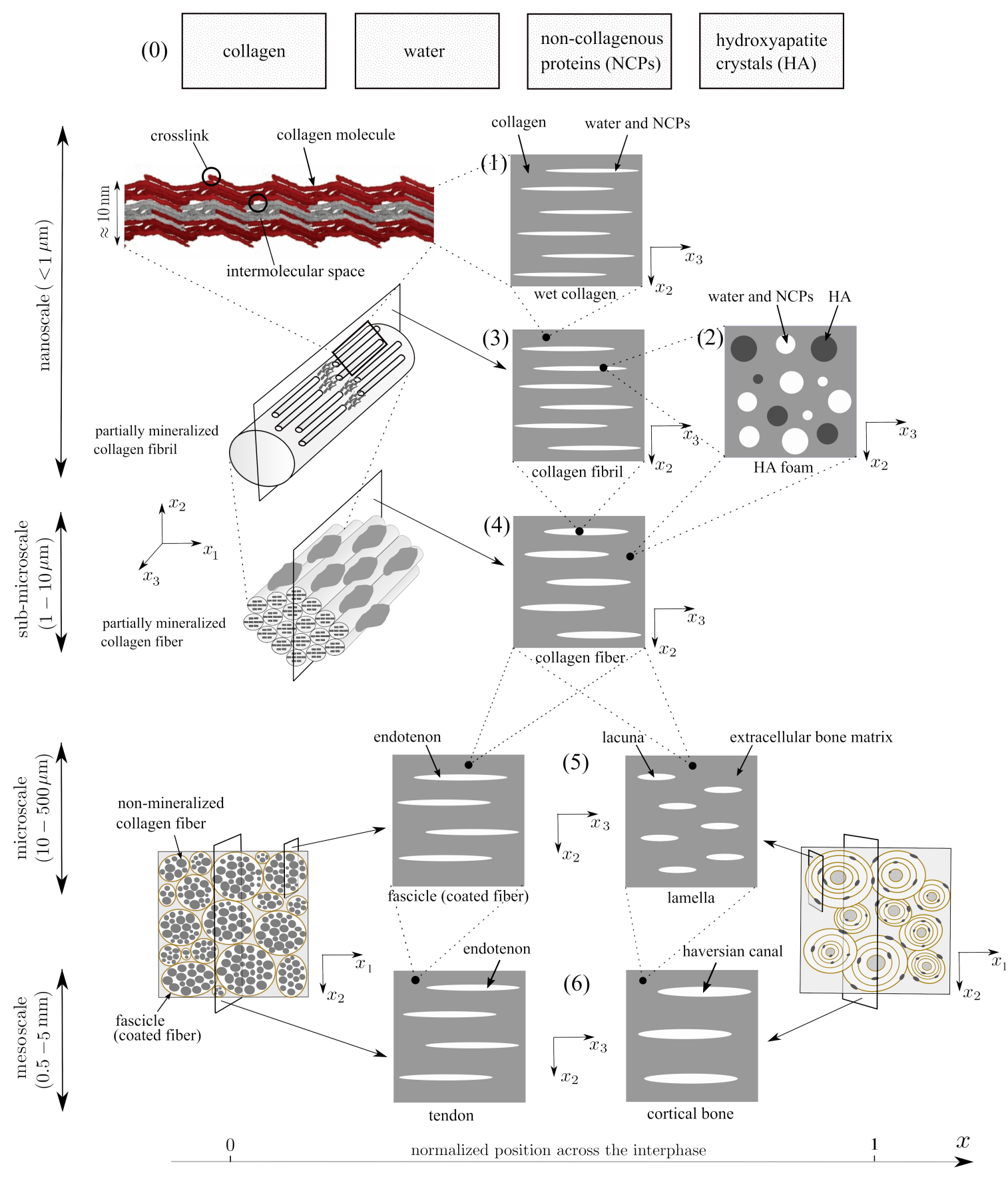

Figure 1: Successive steps of our multiscale modeling approach: (0) elementary nanoscale components; (1) wet collagen composite formed by a collagen molecules matrix containing water and NCPs-filled pores (image adapted from [35]); (2) HA foam formed by HA crystals containing water and NCPs-filled pores; (3) partially mineralized collagen fibril made up from wet collagen and intrafibrillar HA foam; (4) partially mineralized collagen fiber formed by partially mineralized collagen fibrils and extrafibrillar HA foam; (5) lamella and unit fascicle built up from a partially mineralized collagen fiber matrix holding lacunae cavities and endotenon, respectively; and (6) cortical bone and tendon formed by a continuous bone matrix (extravascular bone) and a bundle of parallel fascicles matrix containing haversian canals and endotenon, respectively. 


\section{A. Nanostructural level}

At the nanostructural level, collagen molecules are attached to each other by crosslinks, which transfer the stresses from one collagen molecule to another [35]. Water initially fills the spaces between collagen molecules. During the mineralization process, mineral crystals are nucleated in the gap zones, thus progressively replacing the water. They then further grow into the overlap zones during development and may be distributed throughout the collagen molecules to form a partially mineralized collagen fibril [36]. To mimic this physiological process, three homogenization steps were used to model the enthesis at the nanostructural level: (1) combining collagen together with water and NCPs, (2) combining HA crystals with water and NCPs, and (3) combining the collagen composite from step 1 with the HA foam from step 2 to form a partially mineralized collagen fibril.

\section{Step 1: wet collagen composite}

The wet collagen composite was modeled as a contiguous cross-linked collagen molecules matrix with holes hosting water and NCPs, where the holes were considered as cylindrical inclusions $[33,37]$. Based on the assumption that a continuum approach can be applied at the nanostructural level, the Mori-Tanaka scheme was used to estimate the effective stiffness tensor of the composite, $\mathbb{C}_{\mathrm{wc}}$, at this scale as

$$
\begin{aligned}
\mathbb{C}_{\mathrm{wc}} & =\left(1-f_{\mathrm{wp}}\right) \mathbb{C}_{\mathrm{col}}+f_{\mathrm{wp}} \mathbb{C}_{\mathrm{wp}}:\left[\mathbb{I}+\mathbb{P}_{\mathrm{wp}}^{\mathrm{col}}\left(\mathbb{C}_{\mathrm{wp}}-\mathbb{C}_{\mathrm{col}}\right)\right]^{-1} \\
& :\left\{\left(1-f_{\mathrm{wp}}\right) \mathbb{I}+f_{\mathrm{wp}}\left[\mathbb{I}+\mathbb{P}_{\mathrm{wp}}^{\mathrm{col}}:\left(\mathbb{C}_{\mathrm{wp}}-\mathbb{C}_{\mathrm{col}}\right)\right]^{-1}\right\}^{-1},
\end{aligned}
$$

where subscripts "wc", "col" and "wp" are referred to the wet collagen composite, collagen, and water-protein mixture, respectively.

\section{Step 2: hydroxyapatite foam}

At the scale of a few hundred nanometers, HA crystals were assumed to be interpenetrated by intercrystalline space filled with water and NCPs [19]. Since no well-defined matrix phase can be identified at this scale, it results convenient to apply the self-consistent scheme to estimate the effective stiffness tensor of the HA foam, $\mathbb{C}_{\mathrm{Hw}}(x)$, which depends upon the mineral gradient across the insertion. Both the mineral phase and the water-protein mixture 
were here assumed to be spherical in shape with a linear elastic and isotropic behavior. Hence,

$$
\mathbb{C}_{\mathrm{Hw}}(x)=\sum_{r=1}^{2} f_{r}(x) \mathbb{C}_{r}:\left[\mathbb{I}+\mathbb{P}_{r}^{0}:\left(\mathbb{C}_{r}-\mathbb{C}_{\mathrm{Hw}}(x)\right)\right]^{-1}:\left\{\sum_{s=1}^{2} f_{s}(x)\left[\mathbb{I}+\mathbb{P}_{s}^{0}:\left(\mathbb{C}_{s}-\mathbb{C}_{\mathrm{Hw}}(x)\right)\right]^{-1}\right\}^{-1},
$$

with $r, s \in$ [HA, wp]. The subscript "Hw" denotes the HA foam. The detailed calculation of the stiffness coefficients is provided in Appendix B.

\section{Step 3: partially mineralized collagen fibril}

The collagen fibril structure was modeled as a composite consisting of a wet collagen composite matrix reinforced with aligned needle-shaped interfibrillar minerals, which were assumed to be distributed along the main axis of the collagen fibril [38]. Again, the classical Mori-Tanaka method was applied to determine the effective stiffness tensor of a partially mineralized collagen fibril, $\mathbb{C}_{\text {fib }}(x)$, as

$$
\begin{aligned}
\mathbb{C}_{\mathrm{fib}}(x) & =\left(1-f_{\mathrm{Hw}}\right) \mathbb{C}_{\mathrm{wc}}+f_{\mathrm{Hw}} \mathbb{C}_{\mathrm{Hw}}(x):\left[\mathbb{I}+\mathbb{P}_{\mathrm{Hw}}^{\mathrm{wc}}:\left(\mathbb{C}_{\mathrm{Hw}}(x)-\mathbb{C}_{\mathrm{wc}}\right)\right]^{-1} \\
& :\left\{\left(1-f_{\mathrm{Hw}}\right) \mathbb{I}+f_{\mathrm{Hw}}\left[\mathbb{I}+\mathbb{P}_{\mathrm{Hw}}^{\mathrm{wc}}:\left(\mathbb{C}_{\mathrm{Hw}}(x)-\mathbb{C}_{\mathrm{wc}}\right)\right]^{-1}\right\}^{-1},
\end{aligned}
$$

where $\mathbb{C}_{\mathrm{wc}}$ and $\mathbb{C}_{\mathrm{Hw}}(x)$ are the effective stiffness tensors resulting from the application of steps 1 and 2, respectively.

\section{B. Sub-microstructural level - partially mineralized collagen fiber}

At the scale of several micrometers (step 4), randomly dispersed extrafibrillar minerals with different sizes and shapes strongly adhere to the outer parts of the collagen fibrils [34]. During the mineralization, these extrafibrillar minerals are located around the fibril surface and then start growing along the main fibril axis, therefore forming a sort of reinforcing structure around the fibril. Consequently, a partially mineralized collagen fiber can be modeled as an extrafibrillar HA foam matrix pervaded by a cylindrical collagen fibril inclusion. In this way, the effective stiffness tensor of the partially mineralized collagen fiber, $\mathbb{C}_{\mathrm{fbr}}(x)$, can be 
estimated using the Mori-Tanaka scheme as

$$
\begin{aligned}
\mathbb{C}_{\mathrm{fbr}}(x) & =\left(1-f_{\mathrm{fib}}\right) \mathbb{C}_{\mathrm{Hw}}(x)+f_{\mathrm{fib}} \mathbb{C}_{\mathrm{fib}}(x):\left[\mathbb{I}+\mathbb{P}_{\mathrm{fib}}^{\mathrm{Hw}}(x):\left(\mathbb{C}_{\mathrm{fib}}(x)-\mathbb{C}_{\mathrm{Hw}}(x)\right)\right]^{-1} \\
& :\left\{\left(1-f_{\mathrm{fib}}\right) \mathbb{I}+f_{\mathrm{fib}}\left[\mathbb{I}+\mathbb{P}_{\mathrm{fib}}^{\mathrm{Hw}}(x):\left(\mathbb{C}_{\mathrm{fib}}(x)-\mathbb{C}_{\mathrm{Hw}}(x)\right)\right]^{-1}\right\}^{-1},
\end{aligned}
$$

where $\mathbb{C}_{\mathrm{Hw}}(x)$ and $\mathbb{C}_{\mathrm{fib}}(x)$ are the effective stiffness tensors resulting from the application of steps 2 and 3, respectively.

Moving from the tendon to bone, apart from the gradual increase in mineral content (already accounted for in step 2), the insertion site also shows a gradual decrease in the organization of collagen fibers. At the tendon level, collagen fibers are aligned and parallellyoriented along the $x_{3}$-axis. These then start bending and intercrossing along the insertion, and become less organized near the bone [9]. Consequently, the effective stiffness tensor of an individual partially mineralized collagen fiber, $\mathbb{C}_{\mathrm{fbr}}(x)$, can be related to the microstructural level (i.e., fiber bundle or pattern) by averaging over the fibers orientation at each position $x$ of the insertion. Hence,

$$
\overline{\mathbb{C}}_{\mathrm{fbr}}(x)=\int_{S}\left(\mathbb{R}(\boldsymbol{n}) \mathbb{C}_{\mathrm{fbr}}(x) \mathbb{R}^{\mathrm{T}}(\boldsymbol{n})\right) p(\boldsymbol{n}, s(x)) d S
$$

where the superscript $T$ denotes the transpose operator; $p(\boldsymbol{n}, s(x))$ is an axisymmetric and spherical probability density function for finding a fiber whose axis is parallel to the unit vector $\boldsymbol{n}$, with $s(x)$ the angular deviation at a position $x$ with respect to the mean fiber direction $x_{3} ; \mathbb{R}(\boldsymbol{n})$ rotates a tensor from this coordinate system into the global coordinate system; and $\boldsymbol{n}$ includes all directions over the unit sphere $S$ [22]. Note that the resulting stiffness tensor of a partially mineralized fibers pattern, $\overline{\mathbb{C}}_{\mathrm{fbr}}(x)$, retains the symmetry class of that of an individual collagen fiber, but results more compliant along the $x_{3}$-direction.

\section{Microstructural level - partially mineralized fibers pattern}

At the microstructural level (step 5), the structure and composition of tendon and bone are no longer identical, and therefore lead to a different approach for modeling a partially mineralized fibers pattern. At the tendon level, a bundle of aligned collagen fibers forms a unit fascicle, which represents the basic unit of a tendon. A thin connective tissue called 
endotenon surrounds each collagen fiber and binds the fibers together [39]. Since the selfconsistent and Mori-Tanaka methods are limited to an inclusion associated with a low volume fraction, the interactions between neighboring fibers cannot be neglected anymore [24]. To circumvent this issue, the so-called "inverse Mori-Tanaka" scheme was applied here simply by inverting the matrix and inclusion phases [17]. In this regard, by considering the connected bundle of collagen fibers as a matrix and endotenon as a cylindrical inclusion, the effective stiffness tensor of a unit fascicle can be estimated. At the bone level, a lamella containing cavities filled with osteocytes called lacunae is enclosed by the continuous bone matrix holding a bundle of preferentially oriented collagen fibers. In addition, the main axis of lacunae was assumed to be oriented along the longitudinal direction of a lamella [40]. Therefore, similarly to the tendon region, the effective stiffness tensor of a lamella can be estimated by considering the lacuna as a cylindrical inclusion coated with a continuous bone matrix. Hence, the effective stiffness tensor of a partially mineralized fibers pattern at the microstructural level, $\mathbb{C}_{\text {mic }}(x)$, was estimated by considering $\mathbb{C}^{0} \equiv \mathbb{C}_{\text {end }} \equiv \mathbb{C}_{\text {lac }} \equiv \mathbb{C}_{\mathrm{wp}}$, so that

$$
\begin{aligned}
\mathbb{C}_{\text {mic }}(x) & =f_{\mathrm{fbr}}(x) \overline{\mathbb{C}}_{\mathrm{fbr}}(x)+\left(1-f_{\mathrm{fbr}}(x)\right) \mathbb{C}_{\mathrm{wp}}:\left[\mathbb{I}+\overline{\mathbb{P}}_{\mathrm{wp}}^{\mathrm{fbr}}(x):\left(\mathbb{C}_{\mathrm{wp}}-\overline{\mathbb{C}}_{\mathrm{fbr}}(x)\right)\right]^{-1} \\
& :\left\{f_{\mathrm{fbr}}(x) \mathbb{I}+\left(1-f_{\mathrm{fbr}}(x)\right)\left[\mathbb{I}+\overline{\mathbb{P}}_{\mathrm{wp}}^{\mathrm{fbr}}(x):\left(\mathbb{C}_{\mathrm{wp}}-\overline{\mathbb{C}}_{\mathrm{fbr}}(x)\right)\right]^{-1}\right\}^{-1} .
\end{aligned}
$$

In this way, the main difference between tendon and bone at this scale was accounted for by considering a linearly varying volume fraction across the interphase $\left(i . e ., 1-f_{\mathrm{fbr}}(x)\right.$ with $x \in\{0,1\}$ ) for their corresponding inclusion (endotenon for $x=0$ or lacuna for $x=1$ ).

\section{Mesostructural level - partially mineralized tissue}

Similarly to the microstructural level, a different modeling approach was used for the tendon and bone to account for their differences in structure and composition at the mesostructural level (step 6). On the one hand, a bundle of parallel fascicles with an interfascicular matrix (endotenon) forms the tendon [41]. Considering a bundle of parallel fascicles as a matrix and endotenon as a cylindrical inclusion, the effective stiffness tensor of tendon can be estimated. On the other hand, cortical bone hosts cylindrical pores called haversian canals, which contain the bone's nerve and blood supplies being embedded into the osteonal lamella 
matrix (extravascular bone) [37]. The effective stiffness tensor of cortical bone can thus be estimated by considering haversian canals as a cylindrical inclusion coated with a continuous bone matrix.

As in step 5, the effective stiffness tensors of a partially mineralized tissue, $\mathbb{C}_{\text {meso }}(x)$, can be estimated by considering $\mathbb{C}^{0} \equiv \mathbb{C}_{\text {end }} \equiv \mathbb{C}_{\text {hav }} \equiv \mathbb{C}_{\mathrm{wp}}$,

$$
\begin{aligned}
\mathbb{C}_{\text {meso }}(x) & =f_{\text {mic }}(x) \mathbb{C}_{\text {mic }}(x)+\left(1-f_{\text {mic }}(x)\right) \mathbb{C}_{\mathrm{wp}}:\left[\mathbb{I}+\mathbb{P}_{\mathrm{wp}}^{\text {mic }}(x):\left(\mathbb{C}_{\mathrm{wp}}-\mathbb{C}_{\text {mic }}(x)\right)\right]^{-1} \\
& :\left\{f_{\text {mic }}(x) \mathbb{I}+\left(1-f_{\text {mic }}(x)\right)\left[\mathbb{I}+\mathbb{P}_{\mathrm{wp}}^{\text {mic }}(x):\left(\mathbb{C}_{\mathrm{wp}}-\mathbb{C}_{\text {mic }}(x)\right)\right]^{-1}\right\}^{-1} .
\end{aligned}
$$

Again, the variation across the interphase was here driven by the a linearly varying volume fraction, $1-f_{\text {mic }}(x)$, for their corresponding inclusion only.

\section{Model parameters}

Like for any other hierarchical material, the knowledge of the mechanical properties and volume fractions of the tendon-to-bone insertion's elementary components, along with the competing gradients in collagen fibers organization and mineral content, are required to assess its overall mechanical behavior. A broad range of values for the mechanical properties (e.g., Young's modulus $E$ and Poisson's ratio $\nu$ ) of the nanoscale components has been reported in the literature, based on experimental testing facilities or molecular dynamics (MD) simulations.

Earlier theoretical studies provided values of around $2 \mathrm{GPa}$ for the Young's modulus of collagen, but recent MD simulations reported higher values for the Young's modulus of a single collagen molecule, ranging from 7 to $19 \mathrm{GPa}$ [33]. The Poisson's ratio of collagen has typically been chosen equal to 0.28 to deliver a Poisson's ratio of around 0.35 for the collagen-water composite (recall step 1) $[18,17]$. Furthermore, HA minerals were typically assumed to have a linear elastic and isotropic behavior, with a Young's modulus ranging from 63 to $165 \mathrm{GPa}$, and a Poisson's ratio of around 0.23 [33]. To date, little is known about the mechanical properties of NCPs. Nevertheless, water-NCPs composite has generally been assumed to have isotropic properties with a Young's modulus ranging from 1.3 MPa to $0.7 \mathrm{GPa}$, and a Poisson's ratio ranging from 0.4999 to 0.45 , thus encompassing typical 

input values in our multiscale model is summarized in Tab. 1.

\begin{tabular}{lcc}
\hline Material & Young's modulus, $E(\mathrm{GPa})$ & Poisson's ratio, $\nu(-)$ \\
\hline Collagen molecules & $1.2-9.6$ & $0.23-0.33$ \\
HA minerals & $63-165$ & $0.18-0.28$ \\
Water-NCPs composite & $0.0013-0.7$ & $0.4999-0.45$ \\
\hline
\end{tabular}

Table 1: Mechanical properties range of the nanoscale components used for modeling the mesoscale effective stiffness tensor of the tendon-to-bone insertion in this study.

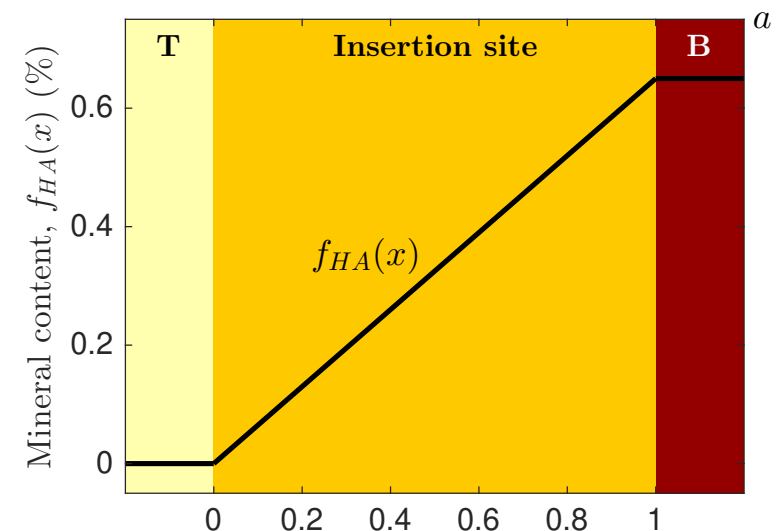

Normalized position along the insertion, $x$

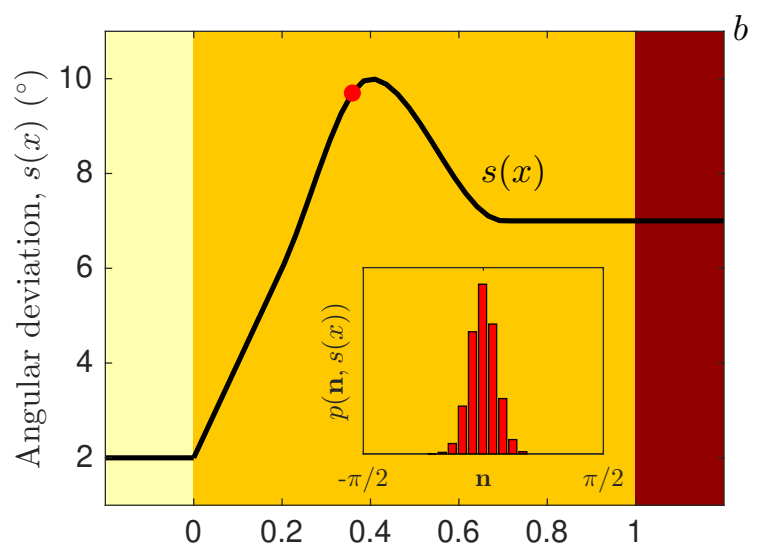

Normalized position along the insertion, $x$

Figure 2: Spatially varying properties of the interphase from the tendon $(\mathrm{T})$ to bone $(\mathrm{B})$ based on competing gradients in (a) mineral content and (b) collagen fibers organization. The insert displays the distribution of collagen fibers near the region of largest fibers disorganization (red dot). These trends are based upon data reported in Refs. [22, 24]. 
The volume fractions of each phase, along with the different homogenization methods applied at each step, are summarized in Tab. 2. At the nanostructural level, the volume fraction for the water-protein mixture, $f_{\mathrm{wp}}$, has been taken according to [17]. At the nanostructural and sub-microstructural levels, we used the values for a mineralization inside and outside the fibrilsas a function of the total mineral content, i.e., $f_{\mathrm{Hw}}$ and $1-f_{\text {fib }}$, following the work of Nikolov and Raabe [17]. In this way, these concurrent variations lead to an overall volume fraction of mineral varying from 0 to $52 \%$ between tendon and bone at the mesoscale, which is in good agreement with values reported in the literature [16, 17]. Furthermore, to account for the difference in structure and composition of the tendon and bone at the micro- and mesostructural levels, volume fractions that vary linearly with respect to the normalized position $x$ across the interphase, i.e., $1-f_{\mathrm{fbr}}(x)$ and $1-f_{\mathrm{mic}}(x)$, have been considered for their respective inclusions at each of these two levels [16, 20].

\begin{tabular}{|c|c|c|c|c|c|c|}
\hline $\begin{array}{l}\text { Hierarchical } \\
\quad \text { levels }\end{array}$ & Steps & $\begin{array}{l}\text { Homogenization } \\
\text { schemes }\end{array}$ & & Phases & Volume fractions (\%) & Relations \\
\hline \multirow{3}{*}{ Nano } & 1 & Mori-Tanaka & $\begin{array}{l}\text { Inclusion } \\
\text { Matrix }\end{array}$ & $\begin{array}{l}\text { Water-NCPs } \\
\text { Collagen }\end{array}$ & $f_{\mathrm{wp}}=35$ & Eq. (2) \\
\hline & 2 & Self-consistent & $\begin{array}{l}\text { Phase } 1 \\
\text { Phase } 2\end{array}$ & $\begin{array}{c}\text { Water-NCPs } \\
\text { HA }\end{array}$ & $f_{\mathrm{HA}}(x)=65 x$ & Eq. (3) \\
\hline & 3 & Mori-Tanaka & $\begin{array}{l}\text { Inclusion } \\
\text { Matrix }\end{array}$ & $\begin{array}{l}\text { Intra-HA foam } \\
\text { Wet collagen }\end{array}$ & $f_{\mathrm{Hw}}=43$ & Eq. (4) \\
\hline Sub-micro & 4 & Mori-Tanaka & $\begin{array}{l}\text { Inclusion } \\
\text { Matrix }\end{array}$ & $\begin{array}{l}\text { Collagen fibril } \\
\text { Extra-HA foam }\end{array}$ & $f_{\text {fib }}=73$ & Eq. (5) \\
\hline Micro & 5 & $\begin{array}{c}\text { Inverse } \\
\text { Mori-Tanaka }\end{array}$ & $\begin{array}{l}\text { Inclusion } \\
\text { Matrix }\end{array}$ & $\begin{array}{l}\text { Endotenon - Lacunae } \\
\text { Collagen fiber }\end{array}$ & $f_{\mathrm{fbr}}(x)=8 x+90$ & Eqs. $(6-7)$ \\
\hline Meso & 6 & $\begin{array}{c}\text { Inverse } \\
\text { Mori-Tanaka }\end{array}$ & $\begin{array}{l}\text { Inclusion } \\
\text { Matrix }\end{array}$ & $\begin{array}{c}\text { Endotenon - Haversian } \\
\text { Fascicle - Lamella }\end{array}$ & $f_{\text {mic }}(x)=8 x+90$ & Eq. (8) \\
\hline
\end{tabular}

Table 2: Volume fractions of each phase along with the different homogenization schemes applied at each step of the multiscale modeling. 

distribution (recall step 4 of Fig. 1).

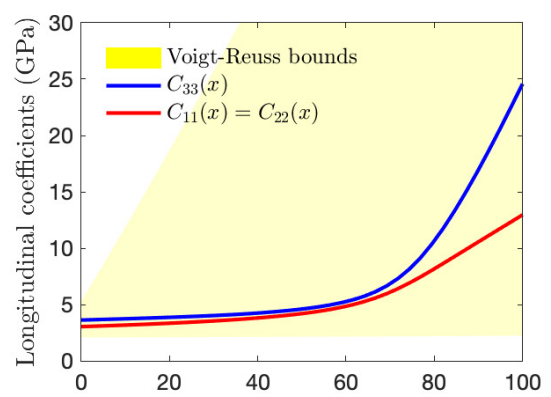

\section{Numerical results}

The effective stiffness properties of the tendon-to-bone insertion from the nanostructural to the mesostructural levels were estimated using the six homogenization steps described in Fig. 1. Three analyses were conducted to rate the performance of the proposed multiscale model. First, several characteristics of the effective stiffness coefficients obtained at the mesostructural level (step 6) were assessed. Second, a parametric study was performed to evaluate the impact of the input parameters on the resulting effective stiffness tensor. Third, an optimization procedure was proposed to identify the model parameters that yielded the best agreement between modeled and measured effective stiffness tensors.

\subsection{Effective stiffness tensor at the mesostructural level, $\mathbb{C}_{\text {meso }}(x)$}

Figure 3 depicts the effective stiffness coefficients of the tendon-to-bone insertion obtained at the mesostructural level, together with the Voigt-Reuss bounds calculated according to [43], using the average mechanical properties from Tab. 1. Note that Voigt notation is adopted to define the coefficients of the fourth-order stiffness tensor and that the $x_{3}$-axis denotes the longitudinal axis, which is aligned with the mean direction of the collagen fibers

Figure 3: Effective stiffness coefficients (in Voigt notation) of the tendon-to-bone insertion obtained at the mesostructural level (continuous lines), along with the Voigt-Reuss bounds (yellow area), using the average mechanical properties from Tab. 1.

First, it is worth pointing out that our model led to a transversely isotropic elastic behavior for the insertion site at the mesoscale, where the stiffness coefficients along the fibers 
(i.e., $C_{33}(x)$ and $\left.C_{44}(x)\right)$ were consistently larger than those normal to the fibers (i.e., $C_{11}(x)$ and $C_{66}(x)$ ), thus retaining the symmetry class of the two surroundings tissues. Recalling that the main constituent properties at the nanoscale were all considered as linear elastic and isotropic, the anisotropy at the different scales was initially triggered by the consideration of geometric features (i.e., the shape of the inclusion) in step 1. Second, the derived effective stiffness tensors at the extremities of the insertion, i.e., $\mathbb{C}_{\text {meso }}(x=0)$ and $\mathbb{C}_{\text {meso }}(x=1)$ from Eq. (8), were in good agreement with experimental values reported in the literature for the Achilles tendon [44, 45] and cortical bone, measured at the tibia mid-diaphysis [46] and femoral diaphysis [47]. Third, the derived stiffness profile across the interphase was nonlinear, displaying a smooth stiffness increase in the non-mineralized fibrocartilaginous region $(\phi(x)<50 \%)$ followed by a sudden rise in stiffness in the mineralized fibrocartilaginous region $(\phi(x)>50 \%)$. The fact that an increase in mineral accumulation within collagen fibers can provide significant stiffening of the enthesis, but only for concentration of minerals above a certain percolation threshold (i.e., $\phi(x) \approx 60 \%$ ), corroborates earlier results and confirms that our model can account for effects of nonuniform mineral accumulation [22]. Finally, it should be noted that the obtained stiffness coefficients all fall within the lower and upper limits defined by the Voigt-Reuss bounds.

\subsection{Parametric study}

The predicted output values of our multiscale model strongly depend on the mechanical properties of the nanoscale components, which showed a rather high dispersion among different studies (recall Tab. 1). We thus performed a parametric study to assess the impact of these input parameters on the values of the resulting effective stiffness tensor at the mesostructural level. The obtained bounds for each of the five independent stiffness coefficients are depicted in Fig. 4. As can be observed, the mechanical properties of the water-NCPs composite inclusion were mostly responsible for relative variations of the shear coefficients (i.e., $C_{44}(x)$ and $\left.C_{66}(x)\right)$ in the low-mineralized region, whereas they barely affected the longitudinal coefficients. The mechanical properties of the collagen molecules matrix significantly affected all stiffness coefficients over the entire tendon-to-bone transition. As expected, the 

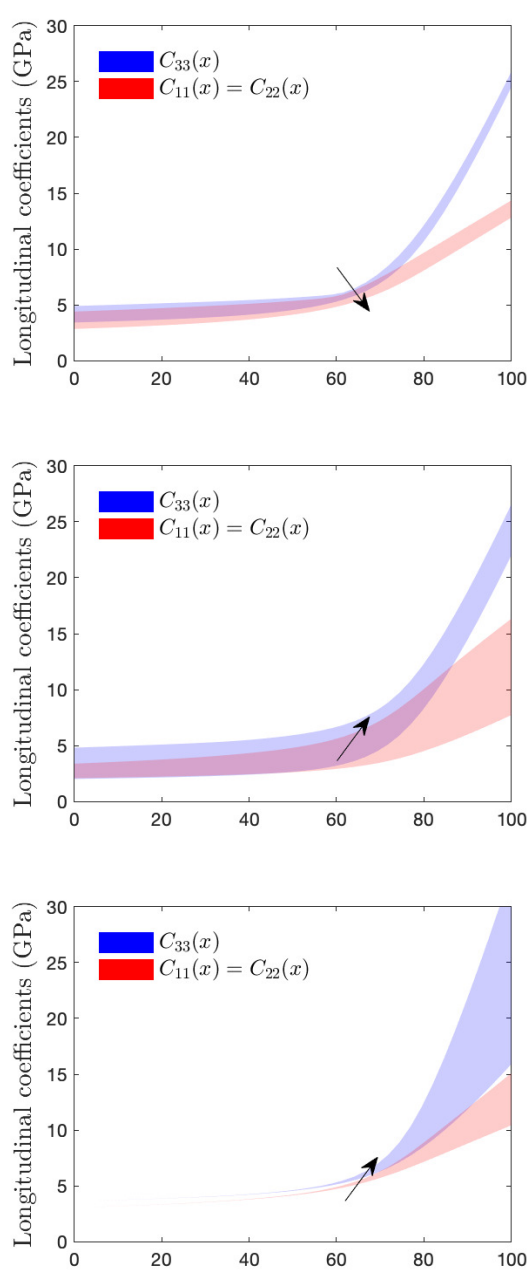
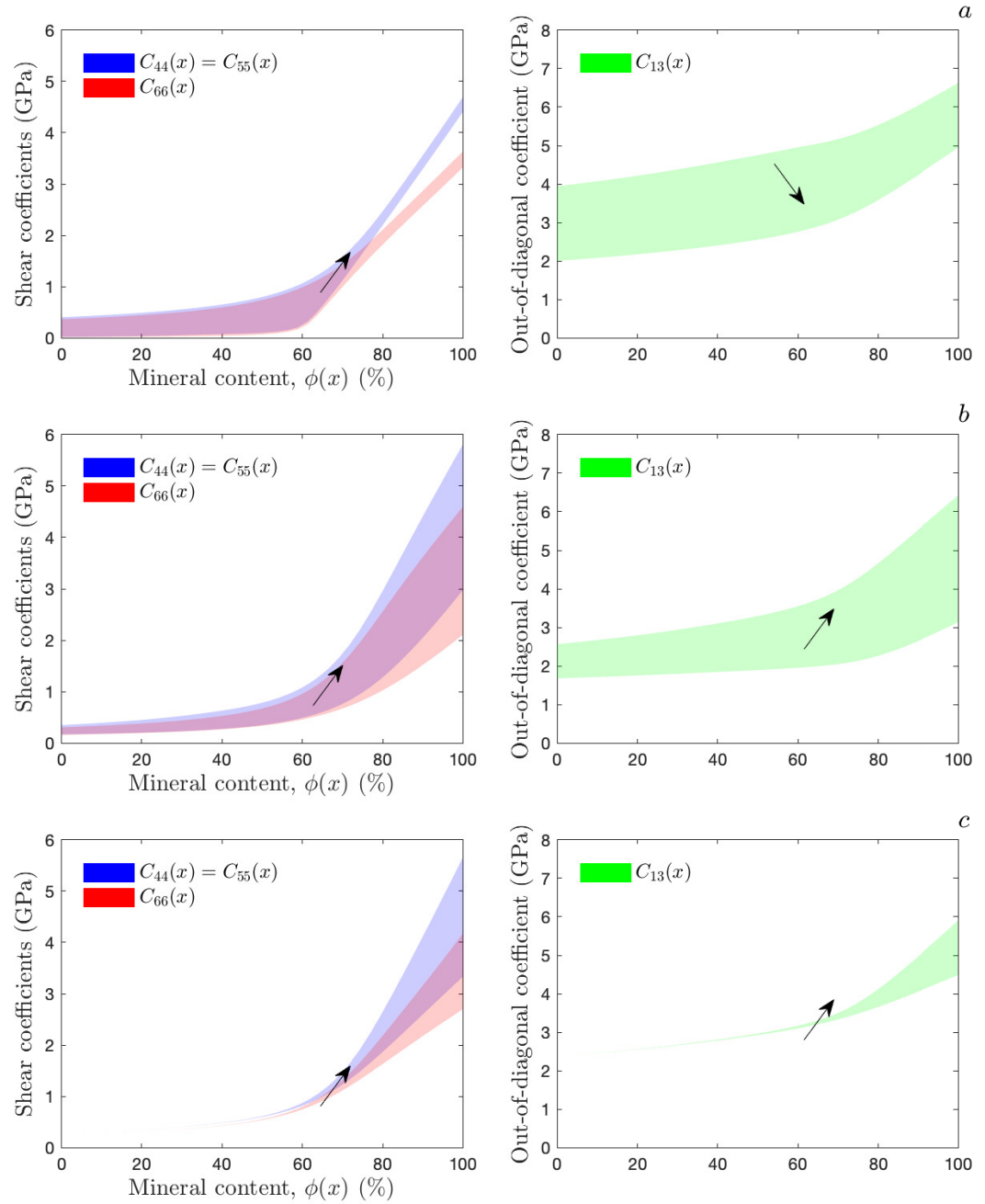

Figure 4: Stiffness coefficients bounds of the tendon-to-bone insertion at the mesostructural level for a variation of the mechanical properties of the nanoscale components: (a) water-NCPs composite; (b) collagen molecules; and (c) HA minerals. Black arrows indicate the resulting trends in stiffness with respect to increasing nanoscale properties.

\subsection{Identification procedure}

To further investigate the sensitivity of the effective stiffness tensor across the interphase, which, in turn, reflects the ability of our model to predict experimentally observed data, we proposed an optimization procedure to identify the model parameters $\boldsymbol{\theta}$ (i.e., the six 
nanoscale properties from Tab. 1) that yielded the best agreement between modeled and measured effective stiffness tensors at the mesostructural scale, denoted by $\mathbb{C}_{\text {meso }}(\boldsymbol{\theta} ; x)$ and $\mathbb{C}^{\exp }(x)$, respectively. Since experimental data for the interphase at the tissue scale are not available in the literature, the optimization was conducted on data from the two surrounding tissues only, i.e., $\mathbb{C}^{\exp }(x=0)$ and $\mathbb{C}^{\exp }(x=1)$, which are summarized in Tab. 3.

\begin{tabular}{|c|c|c|c|c|c|c|c|}
\hline \multirow{2}{*}{ Material } & \multirow{2}{*}{ Position } & \multicolumn{5}{|c|}{ Stiffness tensor $\mathbb{C}^{\exp }(x)$} & \multirow{2}{*}{ Reference } \\
\hline & & $C_{11}^{\exp }$ & $C_{33}^{\exp }$ & $C_{13}^{\exp }$ & $C_{55}^{\exp }$ & $C_{66}^{\exp }$ & \\
\hline Tendon & $x=0$ & 3.08 & 4.51 & 3.10 & 0.04 & 0.02 & [44] \\
\hline Cortical bone & $x=1$ & 14.79 & 26.64 & 6.31 & 5.52 & 3.65 & {$[46]$} \\
\hline
\end{tabular}

Table 3: Reference stiffness coefficients (in GPa) for tendon and bone at the mesostructural scale.

Formally, the optimal model parameters $\hat{\boldsymbol{\theta}}$ result from the minimization of an objective function $F(\boldsymbol{\theta})$ in a least-squares sense as,

$$
\hat{\boldsymbol{\theta}}=\arg \min _{\boldsymbol{\theta} \in \Theta} F(\boldsymbol{\theta}) \quad \text { with } F(\boldsymbol{\theta})=\frac{1}{N} \sum_{n=1}^{N}\left(\frac{C_{n}^{\exp }-C_{n}(\boldsymbol{\theta})}{C_{n}^{\exp }}\right)^{2},
$$

where $\boldsymbol{C}^{\exp }$ and $\boldsymbol{C}(\boldsymbol{\theta})$ are vectors that contain the measured and modeled stiffness coefficients, respectively, $N$ is the number of stiffness coefficients, and $\Theta$ denotes the bounds of the model parameters $\boldsymbol{\theta}$, which were taken according to Tab. 1. Genetic algorithms were applied to solve Eq. (9) owing to their ability in finding a near global solution for non-convex multidimensional objective functions, without the need of an accurate initial guess for the model parameters [48]. For this set of experimental data, the identification procedure delivered the

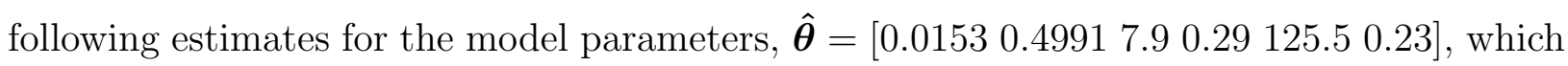
pairwise correspond to the Young's moduli (GPa) and Poisson's ratios of the water-NCPs composite, collagen molecules and HA minerals, respectively. Figure 5 depicts the optimal matching between the modeled and measured effective stiffness tensors at the mesostructural scale. An excellent agreement can be observed between the modeled and measured longitudinal stiffness coefficients, with relative errors that are less than $5 \%$. However, the agreement for the shear and out-of-diagonal stiffness coefficients was moderate for the ten- 

for a stronger anisotropy in the transverse direction.
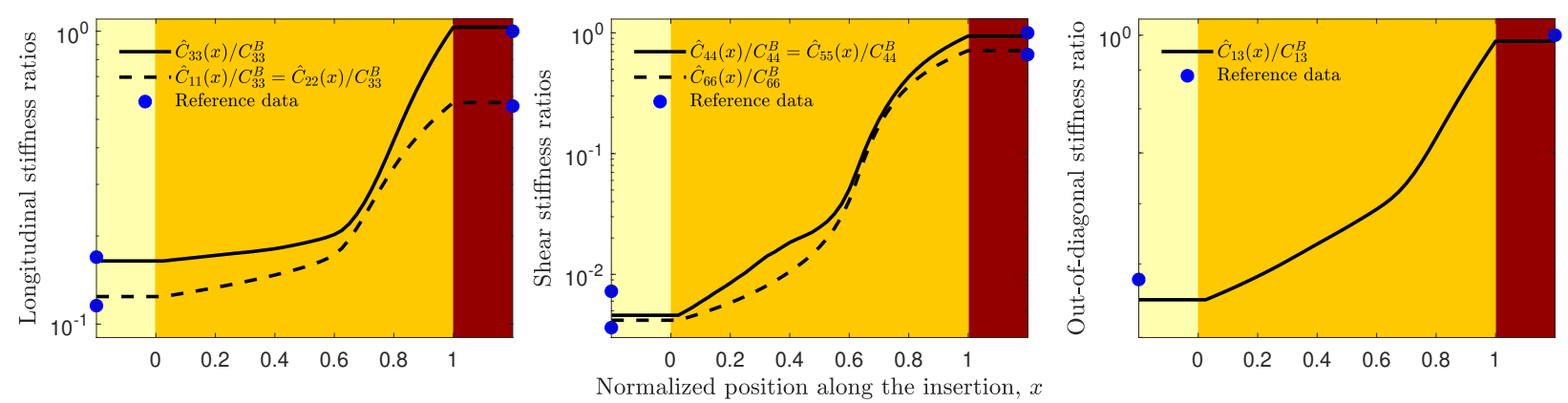

Figure 5: Optimal matching between the modeled and measured effective stiffness tensors at the mesostructural scale. Results were normalized with respect to the stiffness coefficients of bone (Tab. 3).

don region, thus indicating that our model lacks some mechanical features that may account

\section{Discussion}

The tendon-to-bone insertion plays a crucial role in the musculoskeletal system, achieving an effective transfer of mechanical stresses across two tissues displaying a substantial gap in mechanical properties of nearly two orders of magnitude. An accurate modeling accounting for the mutiscale and composite nature of this interphase is essential to deepen our understanding of complex biological interphases and has potential applications both for clinical purposes and for the development of biomimetic strategies in engineering. In this study, we proposed a multiscale model spanning four different hierarchical levels, from the nano- to the mesoscale, connected based on homogeneization procedures. Starting from the mechanical properties of the elementary nanoscale components, this multiscale modeling strategy allowed deriving the effective stiffness tensor of the enthesis at the mesoscale. Since an analytical formulation was used at every step, the computational cost of modeling was very low.

The main findings from this study were as follows: (1) the effective stiffness tensor across the interphase was found to be transversely isotropic at the mesostructural level (Fig. 3), thus retaining the symmetry class of the two surrounding tissues (i.e., tendon and bone); (2) the mechanical properties of the elementary nanoscale components were shown to impact 
the stiffness coefficients of the interphase at the mesoscale (Fig. 4), and were subsequently optimized, by solving an inverse identification procedure, to match available ultrasonic data for the two surroundings tissues (Fig. 5); and (3) our modeling results for the interphase were in qualitative agreement with stochastic finite-element estimates [22, 23] and reported experimental values for the insertional zones of human meniscal attachments into underlying bone [49], thus supporting the hypothesis that the tendon-to-bone insertion can be seen as a continuous functionally graded material.

Indeed, in accordance with these earlier models, our results indicated that the competing gradients in mineral concentration (at the nanoscale) and collagen fibers organization (at the microscale) are important factors in determining the effective mechanical behavior of the tendon-to-bone insertion at the mesoscale. On the one hand, the linear increase in mineral content caused a stiffening of the interphase that became significant beyond a certain percolation threshold. On the other hand, the decreasing collagen fiber organization across the interphase led to a reduced tissue stiffness along the main fibers direction. The existence of a region that is more compliant than either tendon or bone (continuous line in Fig. 6) was the result of these competing gradients. Moreover, it can be shown that the dominant factors driving the width and depth of this compliant region are the onset of mineralization and the angular deviation from the main collagen fibers direction (dashed line in Fig. 6).

Despite these promising results, mismatch observed between the model predictions and experimental data (Fig. 5) could rise from simplifying hypotheses that were adopted at different stages of the modeling. First, the transition between different hierarchies from the nanoscale to the mesoscale is continuous rather than discrete in real biological structures [33]. However, a limited number of length scales was accounted for in the modeling, by assuming the existence of a RVE at each scale and each normalized position across the interphase, in order to fulfill the separation-of-scales requirements (i.e., features at a previous scale are much smaller than those at the next scale). This is not necessarily correct for the tendon-tobone insertion because the features at a previous scale may not be strictly infinitesimal with respect to a larger scale. Although several continuum micromechanics approaches have been used to model partially mineralized tissues with a certain success [16-20, 29, 50], their use 


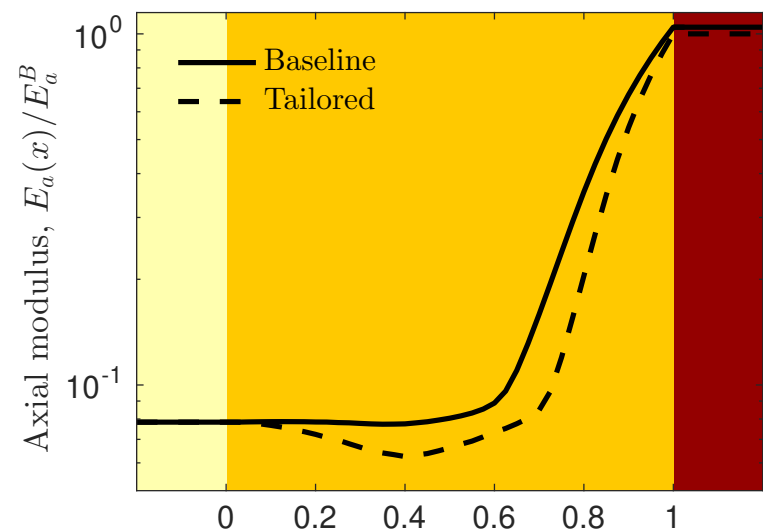

Normalized position along the insertion, $x$

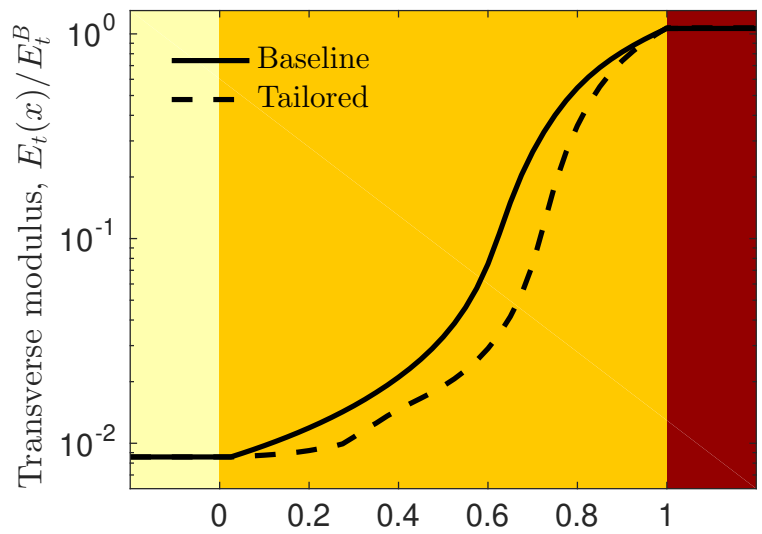

Normalized position along the insertion, $x$

Figure 6: Mesostructural level estimates of the longitudinal elastic moduli. The baseline (continuous line) displays the axial and transverse moduli obtained from the optimal stiffness coefficients depicted in Fig. 5, whereas the tailored case (dashed line) was obtained by delaying the onset of mineralization (to $x \approx 0.2$ ) and reducing the collagen fibers organization (angular deviation increased to $5^{\circ}$ ).

at the nanoscale has sparked important discussions among the homogenization community and is open to debate [51]. For instance, the nano-sized dimensions of mineralized tissue components as well as their spatial arrangements and interactions motivated alternative approaches based on discrete atomistic simulations, specifically molecular dynamics [52]. A second challenging issue was the selection of the mechanical properties of the nanoscale components, i.e., Young's moduli and Poisson's ratios. In this study, all nanoscale components (i.e., water-NCPs composite, collagen molecules and HA crystals) were assumed to have a linear elastic and isotropic behavior. Wide range of values for these nanoscale properties have been reported in the literature (recall Tab. 1) and different choices of such properties may lead to very different results. For instance, atomistic modeling approach of collagen molecules revealed that collagen may have a highly nonlinear viscoelastic behavior [53]. Likewise, the geometry and properties of HA crystals are still subject to debate. This includes the shape of the mineral particles, which have been considered both as needles $[38,54]$ or plates $[5,34]$. Some evidences also shown that HA crystals may be oriented randomly around the mineralized collagen fibrils [55], that their size can change during 
the mineralization from the tendon to bone [56], or that their mechanical properties are anisotropic [57]. Third, only a few studies differentiated between intra- and extrafibrillar mineral volume fractions. Indeed, the mineralization of fibril arrays, in particular the ratio between extra- and intrafibrillar mineral content, has sparked important discussions. Some authors mentioned a high intrafibrillar mineral content $[58,59]$, whereas some others evidenced that the mineral content is mainly located in the extrafibrillar matrix [60,61]. More recent contributions underlined that mineralization pathways in bone are neither exclusively intrafibrillar nor extrafibrillar [62], but rather form a continuous cross-fibrillar phase [38], but this feature remains largely unexplored for the tendon-to-bone insertion [23]. Fourth, the profiles of the competing gradients in mineral content and collagen fibers orientation were selected according to earlier modeling strategies [22, 23], themselves based on Raman spectroscopy [26] and polarized light microscopy [42]. Nevertheless, recent experimental developments for measuring these finely tuned gradients across the interphase are likely to provide different (nonlinear) profiles [63, 64], which could be used as well to feed our modeling approach. Fifth, based on the considered biological phases and their interactions at each scale $[34,56]$, different methods have been used to estimate the effective stiffness tensor at each homogenization step: The Mori-Tanaka method (steps 1, 3 and 4), the selfconsistent method (step 2), and the "inverse Mori-Tanaka" scheme (steps 5 and 6). It is commonly ackowledged that the Mori-Tanaka method shows limited performance for problems involving high volume fraction of inclusions over around 35\% [65], since the inclusions' distribution and interactions are not accounted for properly. Therefore, the attributions of matrix and inclusions roles in our modeling steps 3 and 4 are open to question, so that other homogenization methods could have been used. For instance, a rigorous approach has been proposed for composites containing multiple classes of aligned ellipsoidal inclusions with a relatively high volume fraction [24], although this scheme can violate the Hashin-Shtrikman bounds at low volume fraction for certain anisotropy of the phases. Lastly, our modeling approach contains many steps, each of which should be validated experimentally in the future. To date, little information is available in the literature concerning the experimental multiscale evaluation of the mechanical properties at the tendon-to-bone attachment. Current 
imaging modalities have been used to map the local content of the main constituents across the interphase $[26,63,10]$, but a direct link with the corresponding variations in mechanical properties is somehow still missing. Ongoing developments of experimental techniques for measuring the local mechanical properties of the interphase at different scales, such as nanoscale dynamic mechanical analysis [66] or scanning acoustic microscopy [67], are expected to provide means of comparing our numerically obtained predictions.

All these uncertainties along with some other model parameters ignored in this work, such as the gradient in protein content [68], the different collagen types across the interphase [4] or the unraveling of tendon fibers into smaller interphase fibrils [10], make the multiscale modeling of the tendon-to-bone insertion a challenging problem with much potential for future works. As such, the modeling process proposed here is straightforwardly extendable to account for damage characteristics as a footprint of clinical burden that occur at the tendonto-bone insertion, which may include for instance the multiscale effects of unloading (e.g., stiffening of the HA foam at the nanoscale or fibers misalignment at the microscale) [56]. Besides, such model paves the way to the design of bioinspired bi-materials [69] that display the functionally graded properties of the enthesis, and their characterization using quantitative ultrasound [70, 71].

\section{Conclusion}

We modeled the tendon-to-bone insertion as a hierarchical composite material and predicted its effective anisotropic stiffness tensor at the tissue scale. Our multiscale analysis involved a bottom-up approach, starting from the nanostructural level (partially mineralized collagen fibril) and then moving up the scales through the sub-microstructural level (partially mineralized fiber), the microstructural level (pattern of partially mineralized fibers), up to the mesostructural level (partially mineralized tissue level). Our modeling results supported the hypothesis that the tendon-to-bone insertion can be considered as a continuous functionally graded material with respect to its mineral concentration and collagen fibers organization, thus confirming earlier results obtained using phenomenological models (i.e., stochastic finite element simulations) and experimentally reported trends. 
This work was partially funded by the BEST-AMUS project (IIN program, CNRS-INSIS),

the "Support for research for newly appointed Associate Professors" and the "Bonus Qualité

Recherche" (Faculté des Sciences et Technologie, Université Paris-Est Créteil).

\section{Appendix A. Hill tensor $\mathbb{P}^{0}$}

Appendix A.1. Hill tensor for a cylindrical inclusion in a transversely isotropic medium

The non-zero components of the Hill tensor $\mathbb{P}^{0}$ for a cylindrical inclusion embedded in a transversely isotropic matrix of stiffness $\mathbb{C}^{0}$ are given according to [72] using Voigt notation, $x_{3}$ being the axis of rotational symmetry,

$$
\begin{aligned}
& P_{11}^{0}=\frac{1}{8} \frac{\left(C_{22}^{0}+C_{66}^{0}\right)+2 C_{66}^{0}}{C_{22}^{0} C_{66}^{0}}, \\
& P_{22}^{0}=\frac{1}{8} \frac{\left(C_{22}^{0}+3 C_{66}^{0}\right)}{C_{22}^{0} C_{66}^{0}}, \\
& P_{12}^{0}=\frac{1}{8} \frac{C_{66}^{0}-C_{22}^{0}}{C_{22}^{0} C_{66}^{0}}, \\
& P_{66}^{0}=\frac{1}{2} \frac{C_{22}^{0}+C_{66}^{0}}{C_{22}^{0} C_{66}^{0}}, \\
& P_{44}^{0}=\frac{1}{2 C_{44}^{0}}, \\
& P_{55}^{0}=P_{44}^{0} .
\end{aligned}
$$


Appendix A.2. Hill tensors for a spherical inclusion in an isotropic medium

Considering the case of a spherical inclusion embedded in an isotropic matrix of stiffness $\mathbb{C}^{0}[72]$, the components of the Hill tensor $\mathbb{P}^{0}$ now read as

$$
\begin{aligned}
& P_{11}^{0}=\frac{7 C_{44}^{0}+2 C_{12}^{0}}{15 C_{44}^{0}\left(C_{12}^{0}+2 C_{44}^{0}\right)}, \\
& P_{12}^{0}=\frac{C_{44}^{0}+C_{12}^{0}}{-15 C_{44}^{0}\left(C_{12}^{0}+2 C_{44}^{0}\right)}, \\
& P_{44}^{0}=\frac{2\left(3 C_{12}^{0}+8 C_{44}^{0}\right)}{15 C_{44}^{0}\left(C_{12}^{0}+2 C_{44}^{0}\right)}, \\
& P_{22}^{0}=P_{33}^{0}=P_{11}^{0}, \quad P_{55}^{0}=P_{66}^{0}=P_{44}^{0}, \quad P_{13}^{0}=P_{23}^{0}=P_{12}^{0} .
\end{aligned}
$$

\section{Appendix B. Effective stiffness tensor of the hydroxyapatite foam $\mathbb{C}_{\mathrm{Hw}}(\boldsymbol{x})$}

The self-consistent scheme for two interpenetrating (spherical) inclusion phases with a linear elastic and isotropic behavior can be solved according to [32]. In such a case, the nonlinear system of equations (3) can be substituted by a system of two nonlinear equations (note that the spatial variable $x$ was omitted here for sake of clarity),

$$
\begin{gathered}
\frac{f_{\mathrm{HA}}\left(K_{\mathrm{HA}}-K_{\mathrm{Hw}}\right)}{1+\alpha_{\mathrm{Hw}}\left(K_{\mathrm{HA}}-K_{\mathrm{Hw}}\right) / K_{\mathrm{Hw}}}+\frac{\left(1-f_{\mathrm{HA})}\left(K_{\mathrm{wp}}-K_{\mathrm{Hw}}\right)\right.}{1+\alpha_{\mathrm{Hw}}\left(K_{\mathrm{wp}}-K_{\mathrm{Hw}}\right) / K_{\mathrm{Hw}}}=0, \\
\frac{f_{\mathrm{HA}}\left(G_{\mathrm{HA}}-G_{\mathrm{Hw}}\right)}{1+\beta_{\mathrm{Hw}}\left(G_{\mathrm{HA}}-G_{\mathrm{Hw}}\right) / G_{\mathrm{Hw}}}+\frac{\left(1-f_{\mathrm{HA})}\left(G_{\mathrm{wp}}-G_{\mathrm{Hw}}\right)\right.}{1+\beta_{\mathrm{Hw}}\left(G_{\mathrm{wp}}-G_{\mathrm{Hw}}\right) / G_{\mathrm{Hw}}}=0,
\end{gathered}
$$

where the two unknowns $K_{\mathrm{Hw}}$ and $G_{\mathrm{Hw}}$ denote the bulk and shear moduli of the HA foam, respectively. The parameters $\alpha_{\mathrm{Hw}}$ and $\beta_{\mathrm{Hw}}$ are defined as

$$
\alpha_{\mathrm{Hw}}=\frac{3 K_{\mathrm{Hw}}}{3 K_{\mathrm{Hw}}+4 G_{\mathrm{Hw}}}, \quad \beta_{\mathrm{Hw}}=\frac{6\left(K_{\mathrm{Hw}}+2 G_{\mathrm{Hw}}\right)}{5\left(3 K_{\mathrm{Hw}}+4 G_{\mathrm{Hw}}\right)} .
$$


Solving the aforementioned system yields the following components for the stiffness tensor of the hydroxyapatite foam,

$$
\begin{aligned}
& C_{11}^{\mathrm{Hw}}=K_{\mathrm{Hw}}+\frac{4}{3} G_{\mathrm{Hw}}, \\
& C_{12}^{\mathrm{Hw}}=K_{\mathrm{Hw}}-\frac{2}{3} G_{\mathrm{Hw}}, \\
& C_{44}^{\mathrm{Hw}}=G_{\mathrm{Hw}}, \\
& C_{22}^{\mathrm{Hw}}=C_{33}^{\mathrm{Hw}}=C_{11}^{\mathrm{Hw}}, C_{13}^{\mathrm{Hw}}=C_{23}^{\mathrm{Hw}}=C_{12}^{\mathrm{Hw}}, C_{55}^{\mathrm{Hw}}=C_{66}^{\mathrm{Hw}}=C_{44}^{\mathrm{Hw}} .
\end{aligned}
$$

\section{References}

[1] M Benjamin, T Kumai, S Milz, BM Boszczyk, AA Boszczyk, and JR Ralphs. The skeletal attachment of tendons-tendon 'entheses'. Comp Biochem Physiol A Mol Integr Physiol, 133(4):931-945, 2002.

[2] J Buschmann and GM Bürgisser. Biomechanics of tendons and ligaments: tissue reconstruction and regeneration. Woodhead Publishing, 2017.

[3] J Apostolakos, TJS Durant, CR Dwyer, RP Russell, JH Weinreb, F Alaee, K Beitzel, MB McCarthy, MP Cote, and AD Mazzocca. The enthesis: a review of the tendon-to-bone insertion. Muscles Ligaments Tendons J, 4(3):333, 2014.

[4] AD Waggett, JR Ralphs, APL Kwan, D Woodnutt, and M Benjamin. Characterization of collagens and proteoglycans at the insertion of the human Achilles tendon. Matrix Biol, 16(8):457-470, 1998.

[5] S Weiner and HD Wagner. The material bone: structure-mechanical function relations. Annu Rev Mater Sci, 28(1):271-298, 1998.

[6] HH Lu and S Thomopoulos. Functional attachment of soft tissues to bone: development, healing, and tissue engineering. Annu Rev Biomed Eng, 15:201-226, 2013.

[7] Y Liu, V Birman, C Chen, S Thomopoulos, and GM Genin. Mechanisms of bimaterial attachment at the interface of tendon to bone. J Eng Mater Technol, 133(1):011006, 2011.

[8] Y Hu, V Birman, A Deymier-Black, AG Schwartz, S Thomopoulos, and GM Genin. Stochastic interdigitation as a toughening mechanism at the interface between tendon and bone. Biophys J, 108(2):431-437, 2015.

[9] SF Tellado, ER Balmayor, and M Van Griensven. Strategies to engineer tendon/ligament-to-bone interface: Biomaterials, cells and growth factors. Adv Drug Deliv Rev, 94:126-140, 2015.

[10] L Rossetti, LA Kuntz, E Kunold, J Schock, KW Müller, H Grabmayr, J Stolberg-Stolberg, F Pfeiffer, SA Sieber, R Burgkart, and AR Bausch. The microstructure and micromechanics of the tendon-bone insertion. Nat Mater, 16(6):664, 2017. 
[11] GM Genin and S Thomopoulos. The tendon-to-bone attachment: Unification through disarray. Nat Mater, 16(6):607, 2017.

[12] N Felsenthal and E Zelzer. Mechanical regulation of musculoskeletal system development. Development, 144(23):4271-4283, 2017.

[13] AG Schwartz, JH Lipner, JD Pasteris, GM Genin, and S Thomopoulos. Muscle loading is necessary for the formation of a functional tendon enthesis. Bone, 55(1):44-51, 2013.

[14] F Saadat, AC Deymier, V Birman, S Thomopoulos, and GM Genin. The concentration of stress at the rotator cuff tendon-to-bone attachment site is conserved across species. J Mech Behav Biomed Mater, 62:24-32, 2016.

[15] GS Jung and MJ Buehler. Multiscale modeling of muscular-skeletal systems. Annu Rev Biomed Eng, 19:435-457, 2017.

[16] A Fritsch and C Hellmich. 'Universal' microstructural patterns in cortical and trabecular, extracellular and extravascular bone materials: micromechanics-based prediction of anisotropic elasticity. J Theor Biol, 244(4):597-620, 2007.

[17] S Nikolov and D Raabe. Hierarchical modeling of the elastic properties of bone at submicron scales: the role of extrafibrillar mineralization. Biophys J, 94(11):4220-4232, 2008.

[18] YJ Yoon and SC Cowin. The estimated elastic constants for a single bone osteonal lamella. Biomech Model Mechanobiol, 7(1):1-11, 2008.

[19] E Hamed, I Jasiuk, A Yoo, Y Lee, and T Liszka. Multi-scale modelling of elastic moduli of trabecular bone. J R Soc Interface, 9(72):1654-1673, 2012.

[20] S Tiburtius, S Schrof, F Molnár, P Varga, F Peyrin, Q Grimal, K Raum, and A Gerisch. On the elastic properties of mineralized turkey leg tendon tissue: multiscale model and experiment. Biomech Model Mechanobiol, 13(5):1003-1023, 2014.

[21] EM Spiesz and PK Zysset. Structure-mechanics relationships in mineralized tendons. J Mech Behav Biomed Mater, 52:72-84, 2015.

[22] GM Genin, A Kent, V Birman, B Wopenka, JD Pasteris, PJ Marquez, and S Thomopoulos. Functional grading of mineral and collagen in the attachment of tendon to bone. Biophys J, 97(4):976-985, 2009.

[23] Y Liu, S Thomopoulos, C Chen, V Birman, MJ Buehler, and GM Genin. Modelling the mechanics of partially mineralized collagen fibrils, fibres and tissue. J R Soc Interface, 11(92):20130835, 2014.

[24] F Saadat, V Birman, S Thomopoulos, and GM Genin. Effective elastic properties of a composite containing multiple types of anisotropic ellipsoidal inclusions, with application to the attachment of tendon to bone. J Mech Phys Solids, 82:367-377, 2015.

[25] AC Deymier, Y An, JJ Boyle, AG Schwartz, V Birman, GM Genin, S Thomopoulos, and AH Barber. Micro-mechanical properties of the tendon-to-bone attachment. Acta Biomater, 56:25-35, 2017. 
[26] B Wopenka, A Kent, JD Pasteris, Y Yoon, and S Thomopoulos. The tendon-to-bone transition of the rotator cuff: a preliminary raman spectroscopic study documenting the gradual mineralization across the insertion in rat tissue samples. Appl Spectrosc, 62(12):1285-1294, 2008.

[27] A Zaoui. Continuum micromechanics: survey. J Eng Mech, 128(8):808-816, 2002.

[28] JD Eshelby. The determination of the elastic field of an ellipsoidal inclusion, and related problems. Proc R Soc Lond A, 241(1226):376-396, 1957.

[29] D Gagliardi, V Sansalone, C Desceliers, and S Naili. Estimation of the effective bone-elasticity tensor based on $\mu \mathrm{CT}$ imaging by a stochastic model. a multi-method validation. Eur J Mech A-Solid, 69:147$167,2018$.

[30] T Mori and K Tanaka. Average stress in matrix and average elastic energy of materials with misfitting inclusions. Acta Metall, 21(5):571-574, 1973.

[31] R Hill. A self-consistent mechanics of composite materials. J Mech Phys Solids, 13(4):213-222, 1965.

[32] C Hellmich, J-F Barthélémy, and L Dormieux. Mineral-collagen interactions in elasticity of bone ultrastructure-a continuum micromechanics approach. Eur J Mech A Solids, 23(5):783-810, 2004.

[33] E Hamed, Y Lee, and I Jasiuk. Multiscale modeling of elastic properties of cortical bone. Acta Mech, 213(1-2):131-154, 2010.

[34] B Alexander, TL Daulton, GM Genin, J Lipner, JD Pasteris, B Wopenka, and S Thomopoulos. The nanometre-scale physiology of bone: steric modelling and scanning transmission electron microscopy of collagen-mineral structure. J R Soc Interface, 9(73):1774-1786, 2012.

[35] J Orgel, TC Irving, A Miller, and TJ Wess. Microfibrillar structure of type I collagen in situ. Proc Natl Acad Sci, 103(24):9001-9005, 2006.

[36] P Fratzl, HS Gupta, EP Paschalis, and P Roschger. Structure and mechanical quality of the collagenmineral nano-composite in bone. J Mater Chem, 14(14):2115-2123, 2004.

[37] A Fritsch, C Hellmich, and L Dormieux. Ductile sliding between mineral crystals followed by rupture of collagen crosslinks: experimentally supported micromechanical explanation of bone strength. $J$ Theor Biol, 260(2):230-252, 2009.

[38] N Reznikov, M Bilton, L Lari, MM Stevens, and R Kröger. Fractal-like hierarchical organization of bone begins at the nanoscale. Science, 360(6388):eaao2189, 2018.

[39] P Kannus. Structure of the tendon connective tissue. Scand J Med Sci Sports, 10(6):312-320, 2000.

[40] E Hamed, E Novitskaya, J Li, P-Y Chen, I Jasiuk, and J McKittrick. Elastic moduli of untreated, demineralized and deproteinized cortical bone: validation of a theoretical model of bone as an interpenetrating composite material. Acta Biomater, 8(3):1080-1092, 2012.

[41] CT Thorpe, KJ Karunaseelan, J Ng Chieng Hin, GP Riley, HL Birch, PD Clegg, and HRC Screen. Distribution of proteins within different compartments of tendon varies according to tendon type. $J$ 
Anat, 229(3):450-458, 2016.

[42] S Thomopoulos, JP Marquez, B Weinberger, V Birman, and GM Genin. Collagen fiber orientation at the tendon to bone insertion and its influence on stress concentrations. J Biomech, 39(10):1842-1851, 2006.

[43] J Qu and M Cherkaoui. Fundamentals of micromechanics of solids. Wiley Online Library, 2006.

[44] BK Hoffmeister, SM Handley, SA Wickline, and JG Miller. Ultrasonic determination of the anisotropy of Young's modulus of fixed tendon and fixed myocardium. J Acoust Soc Am, 100(6):3933-3940, 1996.

[45] J Brum, M Bernal, JL Gennisson, and M Tanter. In vivo evaluation of the elastic anisotropy of the human Achilles tendon using shear wave dispersion analysis. Phys Med Biol, 59(3):505, 2014.

[46] S Bernard, J Schneider, P Varga, P Laugier, K Raum, and Q Grimal. Elasticity-density and viscoelasticity-density relationships at the tibia mid-diaphysis assessed from resonant ultrasound spectroscopy measurements. Biomech Model Mechan, 15(1):97-109, 2016.

[47] X Cai, H Follet, L Peralta, M Gardegaront, D Farlay, R Gauthier, B Yu, E Gineyts, C Olivier, M Langer, A Gourrier, D Mitton, F Peyrin, Q Grimal, and P Laugier. Anisotropic elastic properties of human femoral cortical bone and relationships with composition and microstructure in elderly. Acta Biomater, 90:254-266, 2019.

[48] N Bochud, J Laurent, F Bruno, D Royer, and C Prada. Towards real-time assessment of anisotropic plate properties using elastic guided waves. J Acoust Soc Am, 143(2):1138-1147, 2018.

[49] KN Hauch, ML Oyen, GM Odegard, and TL Haut Donahue. Nanoindentation of the insertional zones of human meniscal attachments into underlying bone. J Mech Behav Biomed Mater, 2(4):339-347, 2009.

[50] D Gagliardi, S Naili, C Desceliers, and V Sansalone. Tissue mineral density measured at the submillimetre scale can provide reliable statistics of elastic properties of bone matrix. Biomech Model Mechan, 16(6):1885-1910, 2017.

[51] E Hamed and I Jasiuk. Elastic modeling of bone at nanostructural level. Mater Sci Eng R Rep, 73(3-4):27-49, 2012.

[52] MJ Buehler. Molecular nanomechanics of nascent bone: fibrillar toughening by mineralization. Nanotechnology, 18(29):295102, 2007.

[53] A Gautieri, S Vesentini, A Redaelli, and MJ Buehler. Viscoelastic properties of model segments of collagen molecules. Matrix Biol, 31(2):141-149, 2012.

[54] S Von Euw, T-H-C Chan-Chang, C Paquis, G Haye, Band Pehau-Arnaudet, F Babonneau, T Azaïs, and N Nassif. Organization of bone mineral: The role of mineral-water interactions. Geosciences, 8(12):466, 2018.

[55] MA Rubin, I Jasiuk, J Taylor, J Rubin, T Ganey, and RP Apkarian. TEM analysis of the nanostructure 
of normal and osteoporotic human trabecular bone. Bone, 33(3):270-282, 2003.

[56] AC Deymier, AG Schwartz, Z Cai, TL Daulton, JD Pasteris, GM Genin, and S Thomopoulos. The multiscale structural and mechanical effects of mouse supraspinatus muscle unloading on the mature enthesis. Acta Biomater, 83:302-313, 2019.

[57] JL Katz and K Ukraincik. On the anisotropic elastic properties of hydroxyapatite. J Biomech, 4(3):221$227,1971$.

[58] S Weiner and W Traub. Organization of hydroxyapatite crystals within collagen fibrils. FEBS letters, 206(2):262-266, 1986.

[59] N Sasaki and Y Sudoh. X-ray pole figure analysis of apatite crystals and collagen molecules in bone. Calcif Tissue Int, 60(4):361-367, 1997.

[60] N Sasaki, A Tagami, T Goto, M Taniguchi, M Nakata, and K Hikichi. Atomic force microscopic studies on the structure of bovine femoral cortical bone at the collagen fibril-mineral level. J Mater Sci: Mater, 13(3):333-337, 2002.

[61] EA McNally, HP Schwarcz, GA Botton, and AL Arsenault. A model for the ultrastructure of bone based on electron microscopy of ion-milled sections. PLOS one, 7(1):e29258, 2012.

[62] M Georgiadis, R Müller, and P Schneider. Techniques to assess bone ultrastructure organization: orientation and arrangement of mineralized collagen fibrils. J R Soc Interface, 13(119):20160088, 2016.

[63] JP Spalazzi, AL Boskey, N Pleshko, and HH Lu. Quantitative mapping of matrix content and distribution across the ligament-to-bone insertion. PLoS One, 8(9):e74349, 2013.

[64] D Qu, SD Subramony, AL Boskey, N Pleshko, SB Doty, and HH Lu. Compositional mapping of the mature anterior cruciate ligament-to-bone insertion. J Orthop Res, 35(11):2513-2523, 2017.

[65] HM Yin, LZ Sun, and Glaucio H Paulino. Micromechanics-based elastic model for functionally graded materials with particle interactions. Acta Mater, 52(12):3535-3543, 2004.

[66] I Zlotnikov, E Zolotoyabko, and P Fratzl. Nano-scale modulus mapping of biological composite materials: Theory and practice. Prog Mater Sci, 87:292-320, 2017.

[67] S Leicht and K Raum. Acoustic impedance changes in cartilage and subchondral bone due to primary arthrosis. Ultrasonics, 48(6-7):613-620, 2008.

[68] S Thomopoulos, GR Williams, JA Gimbel, M Favata, and LJ Soslowsky. Variation of biomechanical, structural, and compositional properties along the tendon to bone insertion site. J Orthop Res, 21(3):413-419, 2003.

[69] MJ Mirzaali, AH de la Nava, D Gunashekar, M Nouri-Goushki, RPE Veeger, Q Grossman, L Angeloni, MK Ghatkesar, LE Fratila-Apachitei, D Ruffoni, EL Doubrovski, and AA Zadpoor. Mechanics of bioinspired functionally graded soft-hard composites made by multi-material 3D printing. Compos Struct, page 111867, 2020. 
[70] N Bochud, Q Vallet, J-G Minonzio, and P Laugier. Predicting bone strength with ultrasonic guided waves. Sci Rep, 7:43628, 2017.

[71] G Rosi, L Placidi, V-H Nguyen, and S Naili. Wave propagation across a finite heterogeneous interphase modeled as an interface with material properties. Mech Res Commun, 84:43-48, 2017.

[72] AP Suvorov and GJ Dvorak. Rate form of the Eshelby and Hill tensors. Int J Solids Struct, 39(2122):5659-5678, 2002. 